\title{
Knowledge and Attitude of Pregnant Women towards Antenatal Exercise in Erbil City
}

\author{
Warda Hassan Abdullah; Department of Midwifery, College of Nursing, Hawler Medical University, Erbil, Iraq. \\ (Correspondence: warda.abdullah@hmu.edu.krd) \\ Badia Mohammed Najib; Department of Midwifery, College of Nursing, Hawler Medical University, Erbil, Iraq.
}

\begin{abstract}
Background and objective: Antenatal exercises provide many health benefits not only to pregnant mothers, but also to the fetuses. In the absence of medical or obstetrical complications, pregnant women are encouraged to continue and maintain active lifestyles during their pregnancies. The objective of this study is to assess knowledge and attitude of pregnant women towards antenatal exercises.

Methods: A descriptive cross -sectional study was conducted to assess mother's knowledge and attitudes regarding exercise during pregnancy. The convenience sample of the study included 100 pregnant women. The study was conducted in Najdi Heydari Health Care Center in Erbil City / Kurdistan region / Iraq from December 2016 to Feb, 2017. Data were collected by interview with the pregnant women. A questionnaire was constructed to collect the data by researchers. A statistical package for social science (SPSS, version20) was used for data analysis the data. All statistical procedures were tested on a probability of P-value was $\leq 0.05$ significant.

Results: The majority of the study sample had poor overall knowledge regarding antenatal exercises (93\%), Interest in attending antenatal exercise classes $80 \%$, the barrier to attend antenatal exercise $79 \%$ during pregnancy. There was very highly significant relationship between the pregnant women's educational level and overall knowledge regarding exercise during pregnancy.

Conclusion: The knowledge of women about exercise during pregnancy was less than average and women do not believe in the positive effect of exercises during pregnancy. The main reason for poor knowledge was inadequate antenatal exercise education. It is recommended that the apply antenatal exercise education teaching program is important to increase pregnant women's knowledge regarding to exercise during pregnancy and change their believe regarding antenatal exercises.
\end{abstract}

Key Words: Pregnancy, Antenatal exercises, Knowledge, Attitude, Assessment

Received: $30 / 11 / 2018$

Accepted: 27/8/2019

Published: 30/11/2019

\section{INTRODUCTION}

Exercise is essential for pregnant women's health and wellbeing.It is a safe and effective way to gain many physical and mental health benefits, pregnancy is a good time to develop healthy lifestyle habits including regular exercise and good nutrition $[1,2,3,4,5]$. Improving the quantity and quality of information related to physical exercise has the potential to correct 
inaccurate perceptions and confer several benefits on maternal and fetal health. Effective intervention strategies should focus not only on the pregnant woman but also extend to her family, friends and exercise provider $[3,4]$. The American College of Obstetricians and Gynecologists [6] and the American College of Sports Medicine (ACSM) [7] recommend low-impact, moderate-intensity and regular exercises for pregnant women irrespective of their physical fitness level for approximately 30 minutes on most days of the week its necessary to the mother and fetus. Knowledge regarding benefits and contraindications to antenatal exercise significantly influenced the attitude of the women towards exercise in pregnancy. There is sample and consistent evidence that promoting physical activity in women of reproductive age may be a promising approach for the prevention of excessive weight gain, gestational diabetes mellitus and subsequent complications suffered by children born from pregnancies [8]. Therefore, providing the essential training and an emphasis on the benefits of exercise, decreases the worry of pregnant women about probable adverse effects on the fetus and also with emphasis on this fact that specific exercise during pregnancy with the preparation of the pelvic floor muscles and the abdominal, prepares the mother for the easier vaginal delivery and without damage to the fetus, also improve mothers' performance for having physical activity [8.9]. And there is some evidence showed that physical activity during pregnancy is linked with a shorten length of labor and reduce delivery complications during delivery, including a diminished need for cesarean section delivery $[10,11]$ exercises throughout pregnancy it can shorten the duration of labor and reduce delivery complications [12, 13]. Now, information shows that for many communities, activity levels are inadequate and many people have adopted a sedentary lifestyle. Likewise, there is a low level of awareness about exercise during pregnancy. Many previous studies indicated that information about antenatal exercise is inadequate among pregnant women $[14,15,16,17]$.

This preliminary study assessed the pregnant women's knowledge and attitude regarding exercise during pregnancy. For a purpose of developing a prenatal exercises program to be implemented in a group of pregnant women to find out the effect of such program on pregnancy outcome.

\section{METHODS}

Across-sectional, descriptive study was carried out in Erbil City, Kurdistan region, Iraq from December 1, 2016 to February 30,2017 with a convenient sample of 100 pregnant women who attended Najdi Heydari Health Care Center, Kurdistan Region, Iraq and agreed to participate in the research. Women with major psychological diseases excluded from the study. The study was approved by the Ethical Committee in the College of Nursing, Hawler Medical University, Registration No:8, 16/ 3/2016 and Directorate of Health No 13203, 25/10/2016, Erbil City. The purpose of the study was explained to all subjects in the study, then informed verbal consents were taken during a researcher interview with the pregnant women.,. Data were collected through interview with pregnant women. A questionnaire was constructed to collect the data by researchers, based on the recommendations of antenatal exercises by American College of Obstetrics and Gynecologists,2015 [6] and other literature review, which included the following variables: pregnant women demographic data, pregnant obstetrical history, knowledge of exercise during pregnancy 
and the last part is the pregnant women's attitude about the possibility of the antenatal exercise class during pregnancy period. The response of the knowledge items included two answers $0=$ incorrect and 1 = correct. There were 38 questions related to knowledge about exercise ( 7 items for types of exercise,12 items for benefits, 11 items for contraindication, and finally 8 items for discontinuing of exercise during pregnancy). To measure the content validity of the tool, the questionnaire was presented through a panel of 16 experts. The split-half reliability was determined, measured through computation of Pearson Correlation Coefficient. The Correlation coefficient was $(r=0.779)$. Data were prepared, organized and entered into the computer file; a statistical package for social science (SPSS, version 20) was used for data analysis the data.Data were analyzed through the application of two approaches, which included: Descriptive data analysis approach (Mean, Standard deviation, frequencies and percentage), and inferential statistical analysis. Fisher exact test was used when more than $20 \%$ of the cells of the table have expected count less than 5 . All statistical procedures were tested on a probability of $p$-value was $\leq 0.001$ very highly significant (VHS), $\leq 0.01$ highly significant (HS), $\leq 0.05$ significant $(\mathrm{S})>0.05$ non-significant (NS).

\section{RESULTS}

The results of the study showed that $72 \%$ of mothers attended primary health care center in the second trimester and $28 \%$ in the third trimester of pregnancy. None attended antenatal exercise class during the last pregnancy and $100 \%$ of them their activity was at level one activity (only daily activities). Table 1 Shows sociodemographic characteristics of 100 pregnant women. Regarding age group, the highest percentage $(48 \%)$ of the study sample their age ranged between $20-25$ years and $X \pm S D 28.79 \pm 5.818$ and the majority (39\%) of them had primary school education. In addition, the highest percentage (77\%) of them were housewives and 93\% of the study sample was multigravida

Table 1: Demographic data of the study sample $n=100$

\begin{tabular}{|c|c|c|}
\hline Variables & $\mathbf{F}$ & (\%) \\
\hline \multicolumn{3}{|l|}{ Age (years) } \\
\hline$\leq 20$ & 12 & $(12)$ \\
\hline $21-25$ & 48 & $(48)$ \\
\hline $26-30$ & 34 & (34) \\
\hline $31-35$ & 6 & (6) \\
\hline \multicolumn{3}{|l|}{$M \pm S D(28.79 \pm 5.818)$} \\
\hline \multicolumn{3}{|l|}{ Education level } \\
\hline Illiterate & 18 & $(18)$ \\
\hline Primary & 39 & (39) \\
\hline Secondary & 26 & $(26)$ \\
\hline Institute & 8 & (8) \\
\hline College & 9 & (9) \\
\hline \multicolumn{3}{|l|}{ Occupation } \\
\hline Formal employment & 11 & $(11)$ \\
\hline Privet employment & 9 & (9) \\
\hline Housewife & 77 & (77) \\
\hline Student & 3 & (3) \\
\hline \multicolumn{3}{|l|}{ Gravida } \\
\hline 1 & 3 & (3) \\
\hline $2-4$ & 93 & (93) \\
\hline$\geq 5$ & 4 & (4) \\
\hline
\end{tabular}

Figure 1 shows the highest percentage $(93 \%)$ of the study sample had poor overall knowledge regarding antenatal exercises during pregnancy. 


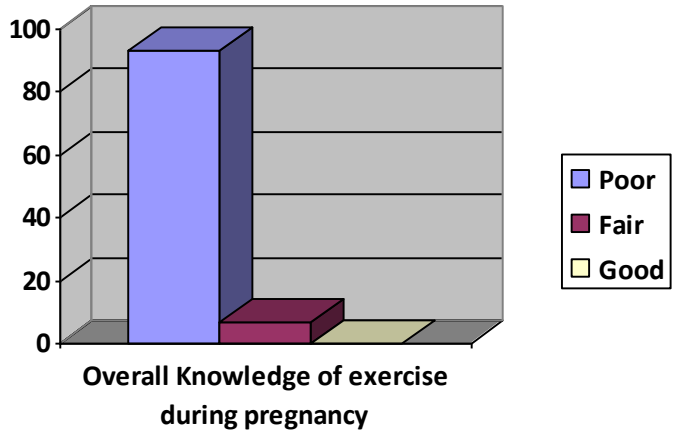

Figure 1: Distribution of the study participants by the level of knowledge regarding antenatal exercises $n=100$

Table 2 shows that the highest percentage (94\%), (94\%), (94\%), (93\%) the respectively of the study sample had poor knowledge regarding the types, benefits, contraindications, discontinuity of exercise during pregnancy. Table 3 shows that 79\%, 80\% of the study sample stated that the barrier to attending antenatal exercise is lack of time and family barriers respectively.

Table 4 indicates the relationship between the age group of the study sample with their overall knowledge regarding antenatal exercise classes during pregnancy, the results of this table show the following: There was no statistically significant relationship between the pregnant women's age with their overall knowledge regarding exercise during pregnancy. $(\mathrm{P}<0.557)$. Table 5 indicates the relationship between the educational level of the study sample with pregnant women overall knowledge regarding antenatal exercise classes during pregnancy, the results of this table show the following: There was a very highly statistically significant relationship between the pregnant women's educational level and overall knowledge regarding exercise during pregnancy, $(P<0.000)$.

Table 2: Pregnant women's knowledge regarding antenatal exercise among study sample $\mathrm{n}=100$

\begin{tabular}{lcccc}
\hline Variables & Good & Fair & Poor & Total \\
& $\mathbf{F}(\%)$ & $\mathbf{F}(\%)$ & $\mathbf{F}(\%)$ & $\mathbf{F}(\%)$ \\
\hline Types of Exercise & $3(3)$ & $3(3)$ & $94(94)$ & $100(100)$ \\
Benefits of Exercise & $0(0)$ & $6(6)$ & $94(94)$ & $100(100)$ \\
Contraindication of Exercise & $5(5)$ & $2(2)$ & $93(93)$ & $100(100)$ \\
Discontinuity of Exercise & $3(3)$ & $3(3)$ & $94(94)$ & $100(100)$ \\
\hline
\end{tabular}

Table 3: Attitude of pregnant women towards antenatal exercises

\begin{tabular}{lllc}
\hline Variables & Yes & No & Total 100 \\
& $\mathbf{F}(\%)$ & $\mathbf{F}(\%)$ & $\mathbf{F}(\%)$ \\
\hline Lack of time & $79(79)$ & $21(21)$ & $100(100)$ \\
Harmful the mother & $14(14)$ & $86(86)$ & $100(100)$ \\
Harmful the Fetus & $14(14)$ & $86(86)$ & $100(100)$ \\
Lack of money for transport & $8(8)$ & $92(92)$ & $100(100)$ \\
Family barriers: & $80(80)$ & $20(200)$ & $100(100)$ \\
Lack health services & $28(28)$ & $72(72)$ & $100(100)$ \\
Feels uncomfortable & $2(2)$ & $98(98)$ & $100(100)$ \\
\hline
\end{tabular}


Table 4: Relationship between age groups of the study sample with pregnant women's overall knowledge regarding antenatal exercises classes $n=100$

\begin{tabular}{lllllll}
\hline Variables & $<20$ & $\mathbf{2 0 - 2 5}$ & $\mathbf{2 6 - 3 0}$ & $\mathbf{3 1 - 3 5}$ & Total & P-Value \\
& $\mathbf{F ( \% )}$ & $\mathbf{F}(\%)$ & $\mathbf{F}(\%)$ & $\mathbf{F}(\%)$ & $\mathbf{F}(\%)$ & \\
\hline Good & $0(0)$ & $0(0)$ & $0(0)$ & $0(0)$ & $0(0)$ & \\
Fair & $0(0)$ & $0(0)$ & $3(3)$ & $1(1)$ & $7(7)$ & $\mathbf{0 . 5 7 7}$ \\
Poor & $12(12)$ & $45(45)$ & $31(31)$ & $5(5)$ & $93(93)$ & \\
Total & $\mathbf{1 2 ( 1 2 )}$ & $\mathbf{4 5 ( 4 5 )}$ & $\mathbf{3 4 ( 3 4 )}$ & $\mathbf{6 ( 6 )}$ & $\mathbf{1 0 0 ( 1 0 0 )}$ & \\
\hline
\end{tabular}

*Fisher-exact test was applied

Table 5: Relationship between the educational level of the study sample with pregnant women's knowledge regarding antenatal exercise class $n=100$

\begin{tabular}{lcccccc}
\hline Variables & Illiterate & Primary & Secondary & Institute \& College & Total & P-Value \\
& $\mathbf{F}(\%)$ & $\mathbf{F}(\%)$ & $\mathbf{F}(\%)$ & $\mathbf{F}(\%)$ & $\mathbf{F}(\%)$ & \\
& & & & & & \\
\hline Good & $0(0)$ & $0(0)$ & $0(0)$ & $0(0)$ & $0(0)$ & \\
Fair & $0(0)$ & $1(1)$ & $0(0)$ & $6(6)$ & $7(7)$ & $<0.001$ \\
Poor & $18(18)$ & $31(31)$ & $26(26)$ & $11(11)$ & $93(93)$ & \\
Total & $\mathbf{1 8 ( 1 8 )}$ & $\mathbf{3 2 ( 3 2 )}$ & $\mathbf{2 6 ( 2 6 )}$ & $\mathbf{1 7 ( 1 7 )}$ & $\mathbf{1 0 0 ( 1 0 0 )}$ & \\
\hline
\end{tabular}

\section{DISCUSSION}

Antenatal exercises improve maternal and fetal health and enhance normal delivery. Prenatal exercise is neglected in the Kurdistan region community. The antenatal exercise program is not conducted in primary health care centers. The results of the present study showed that the activity level of all study samples is at level one activity which means only daily activity. And that might due to the attitudes of a community about exercise in general and during pregnancy specifically. The study showed that knowledge of study sample was very poor as the majority of them had poor overall knowledge and information concerning antenatal exercise, as such services are not available for pregnant women in the government agencies. These results are consistent with the findings of another study
Muhammad (2014) reported that $81.2 \%$ of the study sample had inadequate knowledge regarding the effect of exercise during pregnancy [17] and Wijesiriwardana \& Gunawardena (2015) showed the majority of their study sample had poor knowledge concerning antenatal exercise [16]. pregnant women that involved in this study had poor knowledge about types of antenatal exercises such as walking, leg raising, pelvic floor, and yoga, these results are inconsistent with a study carried out by Chidozie et al. (2014), reported that most of the women had knowledge of pelvic floor exercise, muscle strengthening exercise, and relaxation and breathing exercise as types of antenatal exercises [19]. Knowledge was inadequate concerning benefits of exercise among the study sample as (6\%) of them stated it reduces the risk of back pain, prevents excessive weight gain. This shows that pregnant 
knowledge about antenatal exercises. These results supported by a study done by Chidozie et al (2014) stated most of the women believe that antenatal exercise reduces the risk of back pain and prevents excessive weight gain [19]. Regarding contraindication to antenatal exercise, women in this study had poor knowledge as only (5\%) of them stated that vaginal bleeding, uterine contractions, difficulty in breathing, and presence of back pain during pregnancy are contraindications to exercise during pregnancy. These results are in agreement with the recommendations of the American College of Obstetrician and Gynecologists (2015) and Sujindra et al (2015) [6, 20].Another appealing finding in this study is that they had poor knowledge also regarding discontinuity of exercise the small percentage of them mentioned the following factors for discontinuity exercise during pregnancy: vaginal bleeding, regular painful contraction, and amniotic fluid leakage, these factors are reported as well by American College of Obstetrician and Gynecologists as a guideline to discontinue exercises during pregnancy [6]. The majority of study participants mentioned that exercise is possible during pregnancy. This emphasizes that women are interested to be involved in the antenatal exercise, therefore, it needs to incorporate exercise in prenatal care by health care providers. This result agrees with a study conducted by Watson et al. (2015) and Lovenson et al. (2015), that mentioned $78 \%$ of women agreed that most women can continue their regular exercise during pregnancy [18, 21].Women were asked about their interest of involvements in antenatal exercise classes. In this study, $80 \%$ of pregnant women agreed to attend antenatal exercise classes these results are encouraging to include antenatal exercise in the education program of Pregnant women. The majority of study sample stated the following limitations for attending prenatal exercises lack time 79\%, family barriers and childcare activities $80 \%$. These results are consistent with a study conducted by Khatri et al. (2014), mentioned that most of the study sample they can attend an antenatal education program once weekly for half an hour [22].

Regarding the association between the age of pregnant women and overall knowledge related to exercise during pregnancy was not statistically significant, these results are consistent with findings of other studies, which showed that no statistically significant association between age of pregnant women's and overall knowledge $[15,16,17]$. There was a significant relationship between the level of education and pregnant women's overall exercises knowledge during pregnancy this results is agree with the findings of Loveness et al (in 2014), which reported that were significant associations between adequate knowledge of physical exercise during pregnancy and their education level [21] and also is in agreement with study conducted by Atiya \& Muhammad in 2014 , who showed that level of knowledge about exercise increase with increasing level of education [22]. Chidozie et al. (2015) reported that there was a statistically significant relationship between the level of education and women overall knowledge exercises during pregnancy [1].

\section{CONCLUSION}

Women's knowledge about exercise during pregnancy was less than average and women's responses indicate that they do not believe in the positive effect of exercises during pregnancy. The main reason for poor knowledge was inadequate antenatal exercise education. It is recommended that the antenatal exercise educationteaching program is important to increase pregnant women's knowledge regarding to 
exercise during pregnancy and to change their beliefs regarding antenatal exercise.

\section{ACKNOWLEDGEMENTS}

The authors are thankful for the staff at Najdi Heydari Health Care Center. Maternity and Rizgary Teaching Hospitals, Erbil City, Iraq. My warmest thanks and wish for health go to the sample who participated in this study.

\section{CONFLICT OF INTERESTS}

There is no conflict of interest and sources of financial support for any of the authors.

\section{REFERENCES}

[1] Chidozie EM, Olubukayomi EA, Taofeek OA, Funmilola AF, Monisola OO, Abiola OO, et al. Practice and Pattern of Antenatal and Postnatal Exercise among Nigerian Women. International Journal of Women's Health and Reproduction Sciences.2015;3(2): 93-98.

[2] Clarke PE, Gross H. Women's behaviour, beliefs and information sources about physical exercise in pregnancy. Journal. Midwifery. 2004; (20):133-141

[3] Connolly CH P, Feltz DL, Pivarnik JM. Overcoming Barriers to Physical Activity During Pregnancy and the Postpartum Period. The Potential Impact of Social Support: National Academy of Kinesiology. 2014; 3: 135-148. http://dx.doi.org/10.1123/kr.2013-0009

[4] Katy K, Natasha L, Hezelgrave b, Andrew H Sh. Exercise in pregnancy. Royal College of Obstetricians and Gynaecologists. 2015 DOI: 10.1111/tog. 12228

[5] Moyer C, Jeffrey L, Xiangming F, Linda E. Influence of exercise mode on pregnancy outcomes: ENHANCED by Mom project. BMC Pregnancy and Childbirth. 2015; 15:133

[6] Physical activity and exercise during pregnancy and the postpartum period. the American college of obstetricians and gynecologists-women's health care physician. Obstet Gynecology .2015;126: 135-42
[7] American College of Sports Medicine. Guidelines for exercise testing and prescription. $7^{\text {th }}$ edit. Lippincott Williams \& Wilkins., 2006, Philadelphia, pp.(194-226).

[8] Maryam R, Habibeh M, Parichehr K, Maryam $M$. Effects of education on exercise (physical activity) performance of pregnant women. International Journal of Medical Research \& Health Sciences. 2015; 12:142146.

[9] Prather H, Spitznagle T, Hunt D. Benefits of Exercise During Pregnancy. PM\&RJ. 2012; Vol.4, 11, Source: PubMed

[10] Marit L. B, Anna M S-Riz. Exercise During Pregnancy and Cesarean Delivery. PMC. 2012; 36 (3): 200-207.

[11] Moyer C, May L. Influence of Exercise Mode of Maternal, Fetal, and Neonatal Health Outcomes Cite. Medical Journal Obstetrics Gynecology. 2014;2 (2): 1036.

[12] Price B. B, Amini S, Kappeler K. Exercise in Pregnancy: Effect on Fitness and Obstetric Outcomes-A Randomized Trial. Medicine \& Science in Sports \& Exercise. 2012; 44 (12): 2263-2269.

[13] Haakstad, LA., Bo K. Exercise in pregnant women and birth weight: a randomized controlled trial. BMC Pregnancy and Childbirth. 2011; 11:66

[14] Rachana N, Lizanne P, Vijaya KK, Amitesh N, Suchitra TH, Prasanna M P. Knowledge, Perception, and Attitude of Pregnant Women Towards the Role of Physical Therapy in Antenatal Care: Journal of Health and Allied Sciences. 2015; Vol 14, 4

[15] Rachana N, Simeen V, Chetna G, Vijaya K, Amitsh N, Suchitra Th. Awareness of antenatal exercise among pregnant woman in tertiary care center, Mangalore, India. JAMSCR. 2015; 3 (2): 243-247

[16] Wijesiriwardana WS, Gunawardena NS. Knowledge, attitudes and practices regarding antenatal exercises among pregnant mothers attending De Soyza Maternity Hospital Colombo. Journal of Obstetrics and Gynaecology. 2015;37(4): 65-71

[17] Atiya KM, Muhammad RA. Assessment of Knowledge and Belief of Pregnant Women Attending Maternal and Child Care Unit in Primary Health Center of Sulaimani City Regarding Exercise During Pregnancy. Kirkuk University Journal /Scientific Studies (KUJSS). 2014; 10; 1: 158-172 
[18] Watson E D, Oddie B, Constantinou D. Exercise during pregnancy: knowledge and beliefs of medical practitioners in South Africa: a survey study. BMC Pregnancy and Childbirth. 2015; DOI 10.1186/s12884-015-0690-1

[19] Chidozie EM, Olubukayomi EA, Adebanjo BA, Olujide OA, Olumide OD, Olabisi AA. Knowledge and Attitude of Nigerian Pregnant Women towards Antenatal Exercise: A Cross- Sectional survey. ISRN Obstetrics and Gynecology. Volume, 2014, Article ID 260539,8pages,http:// dx.doi.org/10.1155/2014/260539

[20] Sujindra E, Bupathy A, Suganya A, Praveena $R$. Knowledge, attitude, and practice of exercise during pregnancy among antenatal mothers. International Journal of Educational and Psychological Researches. 2015; Vol 1; Issue 3

[21] Loveness AN, Esther MN, Hastings S. Exercise Practice Among Women Attending Antenatal Care at the University Teaching Hospital in Lusaka, Zambia. Science Journal of Public Health. 2015; 3(3): 361-365

[22] Khatri Ak, Sirohi S, Dixit S, Rai S, pandey D, Effect of antenatal exercise on outcome of labor. National Journal of Community Medicine. 2014; Vol 5, Iss 3 